\title{
Efeito da Oferta de Náuplios de Artemia franciscana Enriquecidos com Ácidos Graxos Poliinsaturados sobre o Desenvolvimento de Pós-Larvas do Camarão Marinho Farfantepenaeus paulensis
}

\author{
Cibele Soares Pontes ${ }^{1}$, Edemar Roberto Andreatta ${ }^{2}$
}

\begin{abstract}
RESUMO - O objetivo deste trabalho foi avaliar a eficácia da técnica de enriquecimento de náuplios de Artemia franciscana, em emulsões de ácidos graxos poliinsaturados (AGP) para a alimentação de pós-larvas do camarão marinho Farfantepenaeus paulensis. Este procedimento visou o acréscimo dos ácidos eicosapentaenóico (EPA) e docosahexaenóico (DHA), essenciais para organismos marinhos, mas deficientes na composição de náuplios provenientes de ecossistemas aquáticos continentais. Os náuplios foram divididos em cinco tratamentos: um grupo controle, composto por indivíduos recém-eclodidos, e quatro grupos enriquecidos cada um com as emulsões Selco, Super Selco, AlgaMac 2000 e emulsão formulada em laboratório à base de óleo de fígado de bacalhau, e então ofertados às póslarvas. Foram comparados os efeitos das dietas quanto à taxa de sobrevivência, ao ganho de peso e à resistência ao estresse nos estádios de $\mathrm{PL}_{10}$ e de $\mathrm{PL}_{20}$. Observou-se que as emulsões Super Selco e Selco foram as que proporcionaram aumento mais destacado dos níveis de EPA e DHA nos náuplios. Entretanto, nenhuma das emulsões aplicadas induziu a diferenças significativas para os parâmetros estudados em comparação com as pós-larvas alimentadas com os náuplios do grupo de controle, indicando que o aumento nos níveis de EPA e DHA não exerceu influência na melhora do desenvolvimento de pós-larvas.
\end{abstract}

Palavras-chave: artemia, bioencapsulação, camarão, farfantepenaeus, nutrição, peneídeos

\section{Effect of Poliunsaturated Fatty Acid Enriched Artemia franciscana Nauplii on Development of Farfantepenaeus paulensis Shrimp Post-Larvae}

\begin{abstract}
The objective of this work was to evaluate the efficiency of Artemia franciscana nauplii, in poliunsturated fatty acid enriched emulsions, for marine shrimp (Farfantepenaeus paulensis) postlarvae feeding. This proceeding aimed the addiction to their diets of eicosapentaenoic acid (EPA) and docosahexaenoic acid (DHA), essential fatty acids for marine organisms, but scarce in nauplii originated in aquatic continental ecosystems. The nauplii were divided in five treatments: a control one with newly-hatched individuals and other four groups, each one enriched with the emulsions: Selco, Super Selco, AlgaMac 2000 and lab made emulsion with cod liver oil, then, offered to the postlarvae. The effect of these diets were compared according to the survival rate, weight gain and stress resistance at the $\mathrm{PL}_{10}$ and $\mathrm{PL}_{20}$ stages. It was observed that Super Selco and Selco emulsions provided the higher increase of the EPA and DHA levels in the nauplii. However, none of the emulsions applied provided significant difference in the observed parameters in comparison to the post-larvae fed with the nauplii from the control group, indicating that the increase of EPA and DHA levels were not critical for the improvement of postlarvae development.
\end{abstract}

Key Words: artemia, bioencapsulation, shrimp, farfantepenaeus, nutrition, penaeidae

\section{Introdução}

O camarão marinho Farfantepenaeus paulensis (Pérez Farfante \& Kensley, 1997) representa importante recurso pesqueiro da região Sul do Brasil, sendo uma das espécies nativas com maior potencial aqüícola. O cultivo larval desta espécie objetiva o repovoamento de lagunas costeiras, bem como o desenvolvimento da carcinicultura nessa região.

Os camarões da família Penaeidae, à qual pertence F. paulensis, são organismos gregários, que se acasalam e desovam nos oceanos, onde ocorre o desenvolvimento larval. Ao atingirem o estágio de pós-larva, migram para os estuários, onde permanecem por toda a fase juvenil, voltando depois ao oceano para a maturação sexual e reprodução. Após o acasalamento, a fêmea libera óvulos que são fertilizados ao passarem pela massa espermática deixada pelo macho em seu télico.

Os ovos eclodem cerca de 12 horas após a liberação, gerando larvas em estágio de náuplio, que se alimentam de reserva vitelínica e empreendem cinco sub-estágios com duração total de aproximada-

\footnotetext{
1 Departamento de Oceanografia e Limnogia, UFRN, Praia de Mãe Luíza, s/n, Via Costeira, Natal, RN, Brasil. CEP: 59014-100. E.mail: cspontes@natal.digi.com.br

2 Professor do Depto. de Aqüicultura, UFSC, Caixa Postal 476. Florianópolis, SC, Brasil. CEP: 88040-900.
} 
mente 36 horas. No estágio seguinte, de protozoéia, as larvas tornam-se fitoplanctófagas, passando por três sub-estágios ao longo de aproximadamente 5 dias. No último estágio larval, denominado misis, apresentam hábito alimentar zooplanctófago, durante três dias, após os quais atingem a fase de pós-larva. Todo esse processo, em condições controladas, pode variar de 12 a 17 dias, de acordo com o manejo alimentar do cultivo (Treece \& Fox, 1993).

As pós-larvas são morfologicamente iguais a um adulto, apresentam hábito bentônico e movimentam-se para frente. Nesta fase, em seu habitat natural apresentam hábito alimentar omnívoro, em condições de cativeiro alimentam-se de ração e biomassa de Artemia adulta (Treece \& Fox, 1993). Tecnicamente, desde a metamorfose até o trigésimo dia, atribui-se cada 24 horas a um estágio pós-larval $\left(\mathrm{PL}_{1}\right.$ a $\left.\mathrm{PL}_{30}\right)$, a partir de então os indivíduos são considerados juvenis.

O passo inicial para o cultivo de larvas de organismos aquáticos ocorreu com a descoberta, por Seale (1933), de que náuplios de Artemia (Leach 1819, Crustacea, Branchiopoda, Anostraca, Artemiidae), constituem uma excelente fonte de alimento vivo para larvas de peixes recém-eclodidos (Sorgeloos, 1980).

A produção comercial intensiva de larvas de peixes e crustáceos é dependente da utilização de alimento vivo, sendo inúmeras as vantagens da utilização do estágio naupliar de Artemia para este fim: alto teor protéico, tamanho adequado, mobilidade e conseqüente aceitação pelo predador, atração organoléptica, carapaça quitinosa fina, facilidade de armazenamento do cisto e praticidade de sua utilização (Sorgeloos, 1980; Léger et al., 1987; Watanabe, 1987; Hontoria et al., 1989).

No entanto, existe grande variabilidade na composição bioquímica dos náuplios, sendo esta diretamente relacionada ao ecossistema em que desenvolveram-se suas biomassas (Watanabe et al., 1978; Vanhaecke \& Sorgeloos, 1983; Léger et al., 1987).

Segundo Sorgeloos \& Léger (1992), a concentração do ácido eicosapentaenóico (EPA) contida nos náuplios de Artemia determina o valor nutritivo dos mesmos para organismos marinhos.

De acordo com Tacon (1987), os lipídeos fornecidos em dietas para crustáceos devem conter os ácidos graxos denominados essenciais, que, por definição, são aqueles que não podem ser sintetizados de novo (a partir de outros compostos) pelo metabolismo intermediário e, ainda assim, são necessários para manutenção da função celular.
Do ponto de vista energético, os lipídeos constituem a mais rica classe de nutrientes, por serem importantes fornecedores de energia metabólica, e possuírem valores superiores de energia bruta $\left(9,5 \mathrm{kcal} . \mathrm{g}^{-1}\right)$. Além de atuarem como fonte de ácidos graxos e outras classes de lipídeos essenciais como os fosfolipídeos, são também importantes na absorção das vitaminas lipossolúveis A, $\mathrm{D}$, E e K, constituem parte da estrutura da membrana celular, além de serem precursores de hormônios esteróides (Tacon, 1987), desempenhando importante papel no metabolismo intermediário e na reprodução de organismos aquáticos (Câmara, 1994).

Os micro-crustáceos do gênero Artemia são classificados em dois grupos de acordo com o seu conteúdo em ácidos graxos poliinsaturados (AGP): o tipo água doce originário de ecossistemas aquáticos continentais e adequado para a alimentação de organismos característicos desses meios; e o tipo marinho proveniente desses ecossistemas e, portanto, próprio para a alimentação de seus organismos (Navarro et al., 1993). Essa classificação baseia-se na premissa de que os organismos de água doce são capazes de converter o ácido linolênico (LNA) em EPA e, então, em docosahexaenóico (DHA), enquanto os marinhos são capazes de converter apenas o EPA em DHA. Além disso, indivíduos do gênero Artemia tipo marinho contêm níveis de EPA superiores aos níveis encontrados no tipo água doce (Watanabe et al., 1978).

Atualmente, a maioria dos cistos de Artemia encontrada no mercado é produzida no lago Great Salt Lake, cidade de Salt Lake, Estado de Utah, Estados Unidos (GSL), pertence à espécie Artemia franciscana e possui como habitat natural ecossistemas aquáticos continentais, sendo, portanto, supostamente inadequados para a alimentação de organismos marinhos. Para suprir a deficiência de ácidos graxos altamente insaturados, característica dos cistos tipo água doce, desenvolveu-se a técnica de bioencapsulação (Watanabe et al., 1980), também conhecida como enriquecimento de Artemia.

Por serem microcrustáceos filtradores não seletivos, os náuplios de Artemia podem ter seus valores nutritivos potencialmente melhorados através da ingestão de enriquecedores naturais ou artificiais. A introdução destes componentes deve ocorrer no estágio de instar II (cerca de oito horas após a eclosão), quando os mesmos abrem seu trato digestivo e tornam-se não seletivos em relação à quantidade $\mathrm{e}$ qualidade das partículas ingeridas.

Bengtson et al. (1991) destacaram as principais 
técnicas de enriquecimento de Artemia. Na técnica britânica, os náuplios são enriquecidos com a microalga Isochrysis galbana, a desvantagem desse método é a obrigatoriedade da manutenção constante do cultivo algal, além da variabilidade da composição de ácidos graxos poliinsaturados (AGP) nas algas. Na técnica japonesa ("método direto"), utiliza-se emulsão de óleo de peixe adicionada a uma mistura metil-ester de $\operatorname{AGP}\left(\mathrm{n}^{-3}\right)$, que, quando ofertada aos náuplios, é ingerida e suas gotículas acumuladas no trato digestivo. Na técnica francesa, utiliza-se uma dieta composta de pó de Spirulina, levedura, aminoácidos, vitaminas, colesterol e óleo de peixe como enriquecedor. Na técnica belga, é utilizada uma mistura auto-dispersante de diferentes fontes de AGP $\left(\mathrm{n}^{-3}\right)$, vitaminas, carotenóides e fosfolipídeos.

Objetivou-se com o presente trabalho avaliar a importância dos AGP para pós-larvas ( $\mathrm{PL}_{1}$ a $\left.\mathrm{PL}_{20}\right)$ do camarão marinho $F$. paulensis, observando-se os efeitos da oferta de náuplios de $A$. franciscana (GSL), enriquecidos com diferentes emulsões ricas dessas substâncias. As respostas quanto à taxa de sobrevivência, ganho de peso e resistência ao estresse foram os parâmetros analisados para avaliação da qualidade das pós-larvas produzidas.

\section{Material e Métodos}

Este trabalho foi desenvolvido no Laboratório de Camarões Marinhos da Universidade Federal de Santa Catarina (Florianópolis - SC, Brasil).

Os camarões utilizados no experimento, que teve duração de 20 dias, foram cultivados de $\mathrm{PL}_{1}$ (peso seco médio de $178,88 \mathrm{mg}$ ) a $\mathrm{PL}_{20}$, distribuídos em densidade populacional de 50/L, em 20 tanques de formato cilindro-cônicos de fibra de vidro de cor preta, com 30 L de água do mar (33\%), sob aeração constante. A água foi trocada diariamente em $100 \%$, e sua temperatura mantida em $25 \pm 1^{\circ} \mathrm{C}$ com o auxílio de um termostato central.

A alimentação, fornecida duas vezes ao dia (10 e 14h), consistiu de náuplios de Artemia franciscana (GSL) obtidos a partir de cistos descapsulados (Sorgeloos et al., 1977), eclodidos sob densidade de $3 \mathrm{~g} / \mathrm{L}$, em água com salinidade de 33\%. Oito horas após a eclosão dos náuplios, tempo necessário para a abertura de seus tratos digestivos, os mesmos foram colocados em emulsões enriquecedoras, diluídas em água do mar (33\%o), cujos componentes, concentração, tempo de enriquecimento e densidade populacional estão demonstrados na Tabela 1. Cada um dos cinco tratamentos teve quatro repetições, cada qual correspondente a um tanque, totalizando 20. Em cada emulsão foram colocados aproximadamente 300.000 indivíduos.

As emulsões Selco, Super Selco e AlgaMac 2000 foram obtidas no comércio e são constituídas, respectivamente, à base de: $200 \mathrm{mg} \cdot \mathrm{g}^{-1}, 450 \mathrm{mg} \cdot \mathrm{g}^{-1}$ de lipídeos tipo marinho (n-3), e pela microalga Schizochytrium liofilizada. A emulsão formulada, produzida em laboratório, foi preparada de acordo com Kontara (1991), à base de óleo de fígado de bacalhau, água destilada e gema de ovo de galinha (na proporção de 5:2:1), preparada diariamente através de homogeneização durante 5 minutos, nas quantidades utilizadas, de modo a evitar a peroxidação dos AGP (Devron, 1992).

Após a bioencapsulação, os náuplios foram lavados em água doce corrente, e ofertados às pós-larvas seguindo critérios quantitativos determinados em estudos previamente realizados no Laboratório de Camarões Marinhos da UFSC para $F$. paulensis. Os indivíduos destinados à análise da composição quími-

\begin{tabular}{|c|c|c|c|}
\hline $\begin{array}{l}\text { Componentes } \\
\text { Components }\end{array}$ & $\begin{array}{l}\text { Concentração }(\mathrm{g} / \mathrm{L}) \\
\text { Concentration }(g / L)\end{array}$ & $\begin{array}{l}\text { Tempo de enriquecimento (horas) } \\
\text { Enrichment time (hours) }\end{array}$ & $\begin{array}{c}\text { Densidade (náuplios/mL) } \\
\text { Density (nauplii/mL) }\end{array}$ \\
\hline $\begin{array}{l}\text { Selco } \\
\text { Selco }\end{array}$ & 0,6 & 24 & 300 \\
\hline $\begin{array}{l}\text { Super Selco } \\
\text { Super Selco }\end{array}$ & 0,6 & 24 & 300 \\
\hline AlgaMac 2000 & 0,4 & 24 & 300 \\
\hline $\begin{array}{l}\text { Emulsão formulada em laboratório } \\
\text { Lab made emulsion }\end{array}$ & 1,0 & 18 & 150 \\
\hline $\begin{array}{l}\text { Náuplios recém-eclodidos } \\
\text { Newly hatched nauplii }\end{array}$ & - & - & - \\
\hline
\end{tabular}

R. Bras. Zootec., v.32, n.6, p.1544-1550, 2003 (Supl. 1) 
ca foram separados com a ajuda de uma tela de nylon de $100 \mathrm{~mm}$ e conservados à temperatura de $20^{\circ} \mathrm{C}$ negativos, para análise posterior da composição de ácidos graxos.

Para extração dos ácidos graxos contidos nos náuplios de Artemia, as amostras foram previamente liofilizadas, em processo de desidratação caracterizado pela sublimação a vácuo da água contida.

Para análise da composição de ácidos graxos, adicionou-se às mesmas uma mistura de padrões internos, de concentração conhecida, composta pelo ácido 13-cis-docosaenóico, alfa-colestano e o hidrocarboneto $\mathrm{C} 36$, dissolvidos em clorofórmio.

As amostras foram analisadas pela técnica de cromatografia gasosa de alta resolução e cromatografia gasosa acoplada à espectrometria de massas, para identificação dos ácidos graxos sob a forma de ésteres metílicos. Todo o procedimento de extração e análise foi conduzido de acordo com Madureira et al. (1995).

A avaliação dos parâmetros estudados ocorreu após as pós-larvas atingirem os estágios de $\mathrm{PL}_{10}$ e $\mathrm{PL}_{20}$.

A taxa de sobrevivência foi calculada através da contagem das pós-larvas vivas coletadas em cinco amostragens de $250 \mathrm{~mL}$ de cada tanque.

O ganho de peso seco (peso final - peso inicial, em $\mathrm{mg}$ ) foi calculado a partir da média aritmética do peso de 30 pós-larvas coletadas aleatoriamente em cada tanque e, em seguida, desidratadas em estufa por $24 \mathrm{~h}$, a $60^{\circ} \mathrm{C}$.

Aplicou-se às pós-larvas teste de resistência a estresse segundo Tackaert et al. (1989) modificado por Coutteau et al. (1996), em que as mesmas foram submetidas a choque osmótico ( $7 \%$ por $1 \mathrm{~h}$ ), tendo retornado em seguida à salinidade de cultivo. Após meia hora de permanência nesse meio, foi contada a quantidade de indivíduos que apresentavam batimento nos pleópodos, atribuindo-se esse padrão à sobrevivência aparente.

Para todos os parâmetros estudados, a análise estatística foi realizada procedendo-se inicialmente a aplicação do teste de normalidade Kolmogorov-Smirnov (aderência) sobre os resultados, percentuais nos casos da sobrevivência e metamorfose, e valores absolutos no caso de ganho de peso. Os dados percentuais foram posteriormente submetidos à transformação em arcoseno para efeito de análise (Steel \& Torrie, 1988). Uma vez verificada a distribuição normal dos dados, realizou-se análise de variância simples (ANOVA), adotando-se um nível de significância $\mathrm{P}<0,05$.

\section{Resultados e Discussão}

O resultado da análise da composição de ácidos graxos dos náuplios enriquecidos com as diferentes emulsões que determinaram os tratamentos está expresso na Tabela 2. Observou-se aumento efetivo do conteúdo de EPA e DHA nos náuplios enriquecidos, com as diferentes emulsões utilizadas em comparação com os náuplios recém-eclodidos. Os náuplios enriquecidos com a emulsão Super Selco apresentaram maiores proporções de EPA e DHA em relação aos demais.

A partir da constatação de que os dados relativos à sobrevivência, ganho de peso e resistência ao estresse apresentaram uma distribuição normal (Kolmogorov-Smirnov), a aplicação de análise de variância (ANOVA) sobre esses parâmetros nos estágios de $\mathrm{PL}_{10}$ e $\mathrm{PL}_{20}$ indicaram a ausência de diferença significativa $(\mathrm{P}<0,05)$ entre os tratamentos (Tabela 3).

A análise química dos náuplios (Tabela 2) mostrou que os indivíduos recém-eclodidos (GSL) não contêm o ácido DHA, o que está de acordo com Navarro et al. (1993). A utilização da emulsão Super Selco induziu ao maior incremento nos níveis de EPA e DHA dos náuplios enriquecidos, em relação aos recém-eclodidos, sendo seguida pela Selco, AlgaMac 2000 e, então, pela emulsão formulada em laboratório.

Diversos autores comprovaram que a bioencapsulação de AGP eleva os níveis de EPA e DHA dos náuplios a serem ofertados a organismos cultivados (Léger et al., 1986; Sorgeloos \& Léger, 1992; Dhont et al., 1993; Barclay \& Zeller, 1996). Os resultados do presente trabalho corroboram registros anteriores de que a composição de náuplios de Artemia é determinada pelo alimento ingerido sendo possível melhorar sua qualidade nutricional através de manipulação dietética.

Rees et al. (1994) testaram comparativamente a alimentação de pós-larvas $\left(\mathrm{PL}_{5}\right.$ a $\left.\mathrm{PL}_{15}\right)$ de Penaeus monodon através de náuplios enriquecidos com Selco em diferentes concentrações (0 a $\left.400 \mathrm{mg} \cdot \mathrm{g}^{-1}\right)$. Foi observado que as maiores taxas de sobrevivência e resistência ao estresse estiveram associadas aos tratamentos correspondentes a concentrações intermediárias, enquanto o maior ganho de peso ocorreu nos indivíduos que receberam a menor faixa de concentração, evidenciando a necessidade de estudos mais precisos em relação à quantidade e qualidade 
Tabela 2 - Freqüência percentual de ácidos graxos dos náuplios de Artemia franciscana (GSL), para a alimentação de pós-larvas do camarão marinho F. paulensis, por componente utilizado na bioencapsulação

Table 2 - Fatty acid composition percentage frequency in Artemia franciscana (GSL) nauplii, for F. paulensis postlarvae feeding, according to the bioencapsulation component

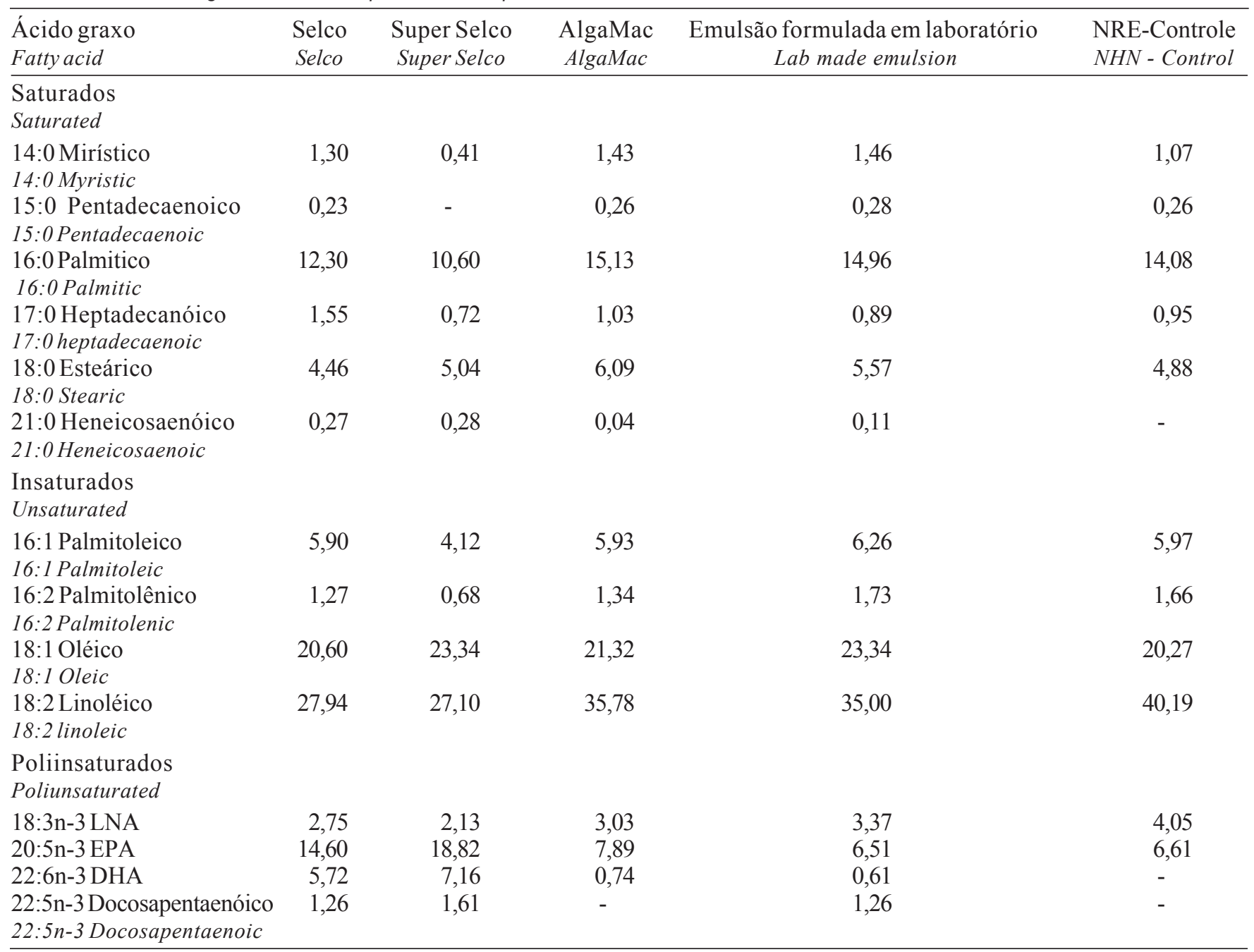

de AGP a serem ofertados. Comparativamente, no presente trabalho, constatou-se que, para pós-larvas de $P$. paulensis, a variação na composição de ácidos graxos das emulsões enriquecedoras utilizadas não foi suficiente para promover diferenças estatisticamente significativas na sobrevivência, ganho de peso e resistência ao estresse das mesmas.

Coutteau et al. (1996) desenvolveram pesquisa nutricional em pós-larvas de Litopenaeus vannamei durante 40 dias, usando diferentes fontes e níveis de EPA e DHA. As taxas de sobrevivência e resistência ao estresse mantiveram-se estatisticamente iguais ao longo de todo o experimento, enquanto os ganhos de peso foram alterados apenas a partir do $18^{\circ}$ dia, afetados pelos níveis de fosfolipídeos. Comparativa- mente, no presente trabalho não se detectaram alterações entre os tratamentos, para os mesmos parâmetros.

Trabalhos realizados com diferentes níveis e fontes de EPA e DHA, na alimentação de diversos estágios de diferentes espécies de camarões, são unânimes em detectar melhoramentos proporcionados pela suplementação de AGP em pelo menos um dos parâmetros estudados. O presente trabalho, embora não tenha encontrado qualquer relação entre o aumento dos níveis de EPA e DHA e a melhoria dos parâmetros de avaliação das pós-larvas, está de acordo com as demais literaturas citadas quanto à ausência de correlação entre os níveis de AGP da dieta e os parâmetros estudados. 


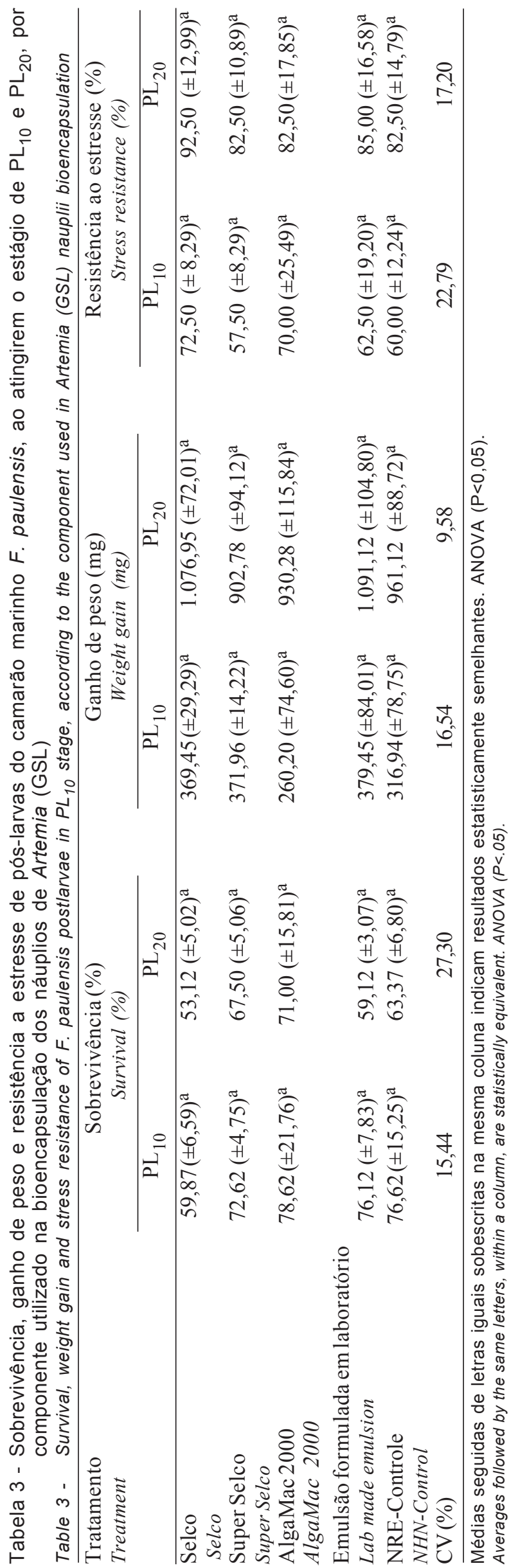

\section{Conclusões}

A utilização da técnica de bioencapsulação empregada proporcionou o aumento dos níveis de ácidos graxos poliinsaturados nos náuplios de Artemia franciscana (GSL).

O incremento dos níveis de ácidos graxos poliinsaturados não exerceu influência sobre a taxa de sobrevivência, o ganho de peso e a resistência ao teste de estresse, das pós-larvas, sendo necessários, portanto, estudos mais aprofundados no sentido de determinar mais detalhadamente as quantidades ideais das concentrações de ácidos graxos a serem utilizadas nas emulsões.

\section{Agradecimento}

Ao Laboratório de Camarões Marinhos da UFSC e toda sua equipe técnica, sem a qual esse trabalho não teria sido possível. Ao Prof. Dr. Luiz Augusto dos Santos Madureira, da Central de Análises Químicas da UFSC, pela extração e análise dos lipídeos contidos nas amostras.

\section{Literatura Citada}

BARCLAY, W.; ZELLER, S. Nutritional enhancement of omega3 and omega - 6 fatty acids in rotifers and Artemia nauplii by feeding spay-dried Schizochitrum sp. Journal of World Aquaculture Society, v.27, n.3, p.314-322, 1996.

BENGTSON, D.A.; LÉGER, P.; SORGELOOS, P. Use of Artemia as a food source for aquaculture. In: CRC Press Inc. (Ed.). Artemia biology. 1.ed. Boca Raton: CRC Press Inc., 1991. v.3, p.255-285.

CÂMARA, M.R. Dietary phosphatidylcoline requirements of Penaeus japonicus BATE and Penaeus vannamei BOONE (Crustacea, Decapoda, Penaeidae). Gent: Universiteit Gent, 1994. 173 p. Tese (Ph.D. in Applied Biological Sciences) - Faculty of Agricultural and Applied Sciences, Universiteit Gent, Belgium, 1994.

COUTTEAU, P.; CÂMARA, M.R.; SORGEloOS, P. The effect of different levels and sources of dietary phosphatidylcholine om the growth, survival, stress resistance, and fatty acid composition of postlarval Penaeus vannamei. Aquaculture, n.147, p.261-273, 1996.

DEVRON, C.A. Marine oils and their effects. Nutrition Reviews, v.50, n.4, p.38-45, 1992.

DHONT, J.; LAVENS, P.; SORGELOOS, P. Preparation and use of Artemia as food for shrimp and prawn larvae. In: Handbook of Mariculture (v.1). In: McVEY, J.P. (Ed.). Boca Raton: CRC Press Inc., 1993. p.61-93.

HONTORIA, F.; NAVARRO, J.C.; VARÓ, I. et al. Artemia cysts in marine larvae cultures: quality evaluation. Aquacultural Engineering, n.8, p.127-138, 1989.

KONTARA, E.K. Growth and survival of tiger shrimp, Penaeus monodon postlarvae fed with Artemia nauplii enriched with 
n-3 highly unsaturated fatty acid. In: LARVI'91 - FISH AND CRUSTACEAN LARVICULTURE SYMPOSIUM. Special Publication European Aquaculture Society, 15., 1991, Amsterdam. Proceedings... Amsterdam, 1991. p.74-75.

LÉGER, P.; BENGTSON, D.A.; SIMPSON, K.L. et al. The use and nutritional value of Artemia as food source. Oceanograph Marine Biology Annual Review, n.24, p.521-623, 1986.

LÉGER, P.; BENGTSON, D.A.; SORGELOOS, P. et al. The nutritional value of Artemia: a review. In: SORGELOOS, P.; BENGTSON, D.A.; DECLEIR, W. et al. (Eds.). Artemia review and its applications. Wetteren: Universa Press, 1987. v. 3, p.1-19.

MADUREIRA, L.A.S.; CONTE, M.H.; EGLINTON, G. Early diagenesis of lipid biomarker compounds in North Atlantic sediments. Paleoceanography, n.10, p.627-642, 1995.

NAVARRO, J.C.; AMAT, F.; SARGENT, J.R. The lipids of the cysts of freshwater - and marine - type Artemia. Aquaculture, n.109, p.327-336, 1993.

PÉREZ FARFAnte, I.; KENSLEY, B. Penaeoid and Sergestoid shrimps and prawns of the world. Key of diagnoses for the families and genera. 1.ed. Paris: Éditions du Muséum National dersquo Histoire Naturale, 1997. 233p.

REES, J. F.; CURÉ, K.; PIYATIRATITÍVORAKUL, S. et al. Highly unsaturated fatty acid requirements of Penaeus monodon postlarvae: an experimental approach on Artemia enrichment. Aquaculture, n.122, p.193-207, 1994.

SEALE, A. Brine shrimp (Artemia) as a satisfactory live food for fishes. American Fisheries Society, n.63, p.129-130, 1933.

SORGELOOS, P.; BOSSUYT, E.; LAVINA, E. et al. Descapsulation of Artemia cysts: a simple technique for the improvment of the use of brine shrimp in aquaculture. Aquaculture, n.12, p.311-315, 1977.

SORGELOOS, P. The use of the brine shrimp Artemia in aquaculture. In: PERSOONE, G.; SORGELOOS, P.; ROELS, O. et al. (Eds). The brine shrimp Artemia. Wetteren: Universa Press, 1980. v.3, p.25-46.

SORGELOOS, P.; LÉGER, P. Improved larviculture outputs of marine fish, shrimp and prawn. Journal of World Aquaculture Society, v.23, n.4, p.12-21, 1992.

STEEL, R.G.D.; TORRIE, J.H. Bioestadística: principios e procedimientos. 2.ed. Cidade do México: McGraw-Hill do México, 1988. 622p.
TACKAERT, W.; ABELIN, P; LÉGER, P. et al. Stress resistance as an criterium to evaluate quality of postlarvae shrimp reared under different feeding procedures. In: SIMPÓSIO BRASILEIRO DE CULTIVO DE CAMARÃO, 3., 1989, João Pessoa. Anais... João Pessoa: MCR Aquacultura, 1989. p.393-403.

TACON, A.G.J. The Nutrition and Feeding of Farmed Fish and Shrimp - A Training Manual 1. The Essential Nutrients. Food and Agriculture Organization of United Nations. Brasília, Brasil. Junho, 1987. 52p.

TREECE, G.D.; FOX, J.M. Design, operation and training manual for an intensive culture shrimp hatchery. 1.ed. Texas: A \& M University Sea Grant College, 1993. 187p.

VANHAECKE, P.; SORGELOOS, P. International Study on Artemia: Hatching data for ten commercial sources of brine shrimp cysts: an re-avaluation of the "hatching efficiency" concept. Aquaculture, n.30, p.43-52, 1983.

WATANABE, T.; OOWA, F.; KITAJIMA, C. et al. Nutritional quality of brine shrimp, Artemia salina as a living feed from the viewpoint of essential fatty acids for fish. Bulletin of Japanese Society of Scientific Fisheries, n.44, p.1115-1121, 1978.

WATANABE, T.; OOWA, F.; KITAJIMA, C. et al. Relationship between dietary value of brine shrimp Artemia salina and their content of w-3 HUFA. Bulletin of Japanese Society of Scientific Fisheries, n.46, p.35-41, 1980.

WATANABE, Y. The use of Artemia in fish and crustacean farming in Japan. In: SORGELOOS, P.; BENGTSON, D.A.; DECLEIR, W. et al. (Eds.) Artemia review and its applications. Wetteren: Universa Press, 1987. v.3, p.373-393. 